\title{
Recent Studies of Proton Drip-Line Nuclei Using the Berkeley Gas-Filled Separator
}

\author{
M. W. Rowe ${ }^{1}$, J. C. Batchelder ${ }^{2}$, V. Ninov ${ }^{1}$, K. E. Gregorich ${ }^{1}$, K. S. Toth ${ }^{3}$, \\ C. R. Bingham ${ }^{3,4}$, A. Piechaczek ${ }^{5}$, X. J. Xu ${ }^{1,6}$, J. Powell $^{1}$, R. Joosten ${ }^{1}$ and \\ Joseph Cerny ${ }^{1,7}$ \\ ${ }^{1}$ Lawrence Berkeley National Laboratory, MS 88, 1 Cyclotron Rd, Berkeley, California 94720 \\ ${ }^{2}$ UNIRIB, Oak Ridge Associated Universities, Oak Ridge, Tennessee 37831 \\ ${ }^{3}$ Department of Physics, Oak Ridge National Laboratory, Oak Ridge, Tennessee 37831 \\ ${ }^{4}$ The University of Tennessee, Knoxville, Tennessee 37996 \\ ${ }^{5}$ Lousiana State University, Baton Rouge, Louisiana 70803 \\ ${ }^{6}$ Institute of Modern Physics, Lanzhou, 730000, China \\ ${ }^{7}$ Department of Chemistry, University of California, Berkeley, CA 94720
}

\begin{abstract}
The Berkeley Gas-filled Separator provides new research opportunities at Lawrence Berkeley National Laboratory's 88-Inch Cyclotron. The use of this apparatus for the study of proton drip-line nuclides is discussed. Preliminary results of ${ }^{78} \mathrm{Kr}$ bombardments of ${ }^{102} \mathrm{Pd}$ targets at mid-target energies of 360,375 and $385 \mathrm{MeV}$ are presented. Improvements planned partially as a result of this measurement are also discussed.
\end{abstract}

\section{INTRODUCTION}

The properties of proton drip-line nuclides have long been a focus of study at Lawrence Berkeley National Laboratory's 88 -Inch Cyclotron. This work includes the confirmation of the first example of direct proton emission, from an isomer of ${ }^{53} \mathrm{Co}$, in 1970 [1]. However, until recently Berkeley has lacked the optimal tools for the study of proton emitters in the region above Sn, where all subsequent examples of direct proton decay have been observed [2]. Virtually all of these later studies have relied on some form of mass separation or analysis to identify the nuclides of interest and reduce unwanted background from other species produced simultaneously. In fact, it is the development of high resolution mass analyzers and velocity filters, as well as high-granularity silicon detector arrays, which has fueled the recent explosion in knowledge of proton drip-line isotopes in the region between $\mathrm{Sn}$ and $\mathrm{Bi}$.

During the Fall of 1998, commissioning of the Berkeley Gas-filled Separator (BGS) began. Although this apparatus was designed primarily as a tool for research on the heaviest elements, its capabilities also make it well suited for the study of proton drip-line isotopes, particularly in the region near lead. After a discussion of the BGS's design and detector systems, preliminary results of the first measurement of very proton-rich nuclides using the BGS will be presented. The primary goal of this experiment was to observe proton emission from ${ }^{177} \mathrm{Tl}[3]$ and ${ }^{176} \mathrm{Tl}$. In the context of 
the results of this measurement, improvements to the BGS which are planned or have already been implemented, will be discussed.

\section{THE BERKELEY GAS-FILLED SEPARATOR}

In order to successfully study proton drip-line nuclei, the experimental apparatus used must meet three requirements. Because such isotopes are produced in low yield, the target must be capable of withstanding large beam currents and/or the transport of the products from the target to the detector system must be very efficient. Second, this transport must be accomplished rapidly, since the half-lives of proton emitters are typically on the order of a few tens of milliseconds or shorter [2]. Finally, there must be some way of identifying the proton-emitter in order to distinguish it from other nuclides produced in much higher yields.

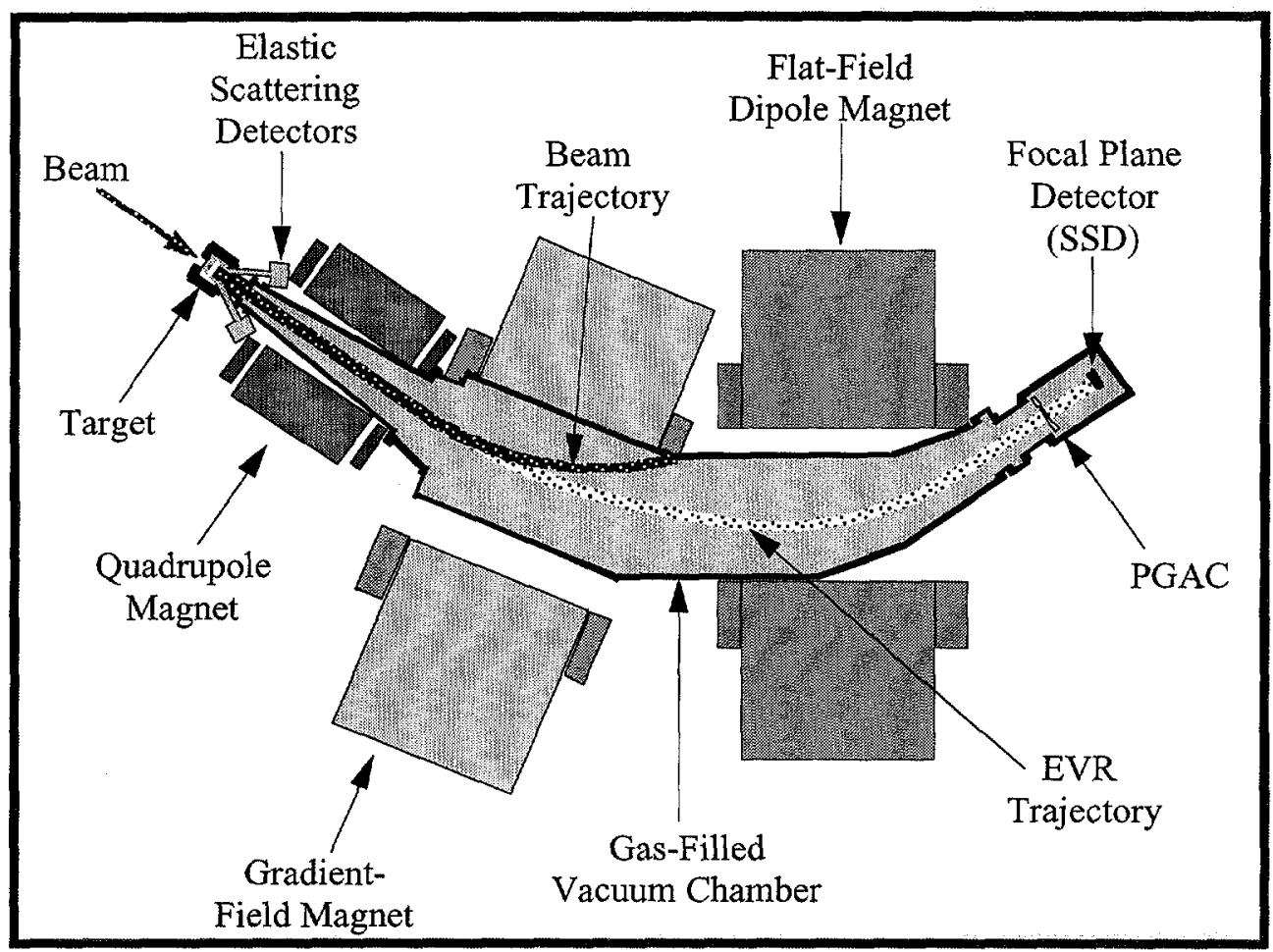

FIGURE 1. A schematic diagram of the Berkeley Gas-filled Separator. The beam enters the apparatus from the left. The detector systems are shown as arranged during the ${ }^{78} \mathrm{Kr}+{ }^{102} \mathrm{Pd}$ measurement described herein. Recent modifications of the system will be described later in this paper.

Gas-filled separators can fulfill these requirements. The residues of fusionevaporation reactions emerge from the target in a variety of charge states. Since the magnetic rigidity, upon which the separation of the various products is based, varies as the inverse of the charge, this can substantially reduce the percentage of a given 
nuclide transported through the separator to the detector system. To reduce this problem, the magnet of a gas-filled separator is filled with a dilute inert gas. According to Bohr's approximation, collisions with the atoms in the gas will leave the reaction products in an average charge state that is proportional to the cube root of their atomic number. To first order, the resulting magnetic rigidity is proportional to the mass over the cube root of the atomic number. This significantly improves the transport efficiency of a gas-filled separator relative to conventional mass analyzers and velocity filters. Unfortunately, the collisions in the gas cause much of the kinematic information to be lost such that the resolution is only sufficient to separate fusion-evaporation residues from incident beam and direct-reaction products. In effect, the gas-filled separator acts as a purifying beam dump. One must rely entirely on the detector system for identification of the implanted nuclides.

A schematic diagram of the Berkeley Gas-filled Separator is shown in Fig. 1. The beam enters from the left through a $100 \mu \mathrm{g} / \mathrm{cm}^{2}$ carbon window. The entire apparatus downstream of this window is filled with $\mathrm{He}$ gas at a pressure of $\sim 1.3 \mathrm{Torr}$. The gas helps to cool the target, which may either consist of a single foil on a target ladder, or a series of foils mounted on a continuously rotating target wheel. Two PIN-diode detectors behind the target monitor beam elastically scattered from the target. Beam and reaction products exit the target and enter a quadrupole magnet followed by a gradient-field dipole magnet that provide vertical and horizontal focussing, respectively. The latter magnet also increases the separation between beam and fusion-evaporation residues, as does the flat-field dipole that follows. The incident beam is bent such that it is dumped between the two dipole magnets. The focal plane of the system is located inside the detector chamber, approximately $1 \mathrm{~m}$ from the exit of the second dipole. The operating parameters of the BGS are presented in Table 1. Note that many of these numbers will depend on the kinematics of the specific reaction employed.

TABLE 1. Operating Parameters of the Berkeley Gas-filled Separator.

\begin{tabular}{lc}
\hline Parameter & Value \\
\hline Maximum beam current: & $5 \times 10^{12}$ ions $/ \mathrm{s}$ \\
Target thickness: & $0.1-1.0 \mathrm{mg} / \mathrm{cm}^{2}$ optimum \\
Angular acceptance: & $\pm 75 \mathrm{mrad}$ horizontal \\
& $\pm 150 \mathrm{mrad}$ vertical \\
Momentum acceptance: & $>50 \%$ \\
Charge acceptance: & $\sim 100 \%$ \\
Transport efficiency: & $20-70 \%$ typical \\
Total Bend: & $70^{\circ}$ \\
Path Length: & $4.6 \mathrm{~m}$ \\
Transport time: & $<2 \mu \mathrm{s}$ \\
Maximum rigidity: & $2.5 \mathrm{~T} \mathrm{~m}$ \\
Overall background rejection: & $>10^{12}$ \\
Dispersion: & $18 \mathrm{~mm} / \% \mathrm{~B} \rho$ \\
Focal plane image size: & $50 \mathrm{~mm}$ vertical \\
\end{tabular}

Inside the detector chamber reaction, products pass through a parallel-grid avalance counter (PGAC) and are subsequently implanted into a $300 \mu \mathrm{m}$-thick single-sided 
silicon strip detector (SSD) located at the focal plane. The SSD is $80 \mathrm{~mm}$ wide by 35 $\mathrm{mm}$ high. It is divided into 16 vertical strips which give crude position sensitivity in the horizontal plane. Signals are measured from both ends of each strip. The sum of these signals is proportional to the implantation or decay energy. Typical resolution for alpha-decay events was $70 \mathrm{keV}$ full-width at half maximum (FWHM). The ratio of either signal to the sum is used to determine the vertical position of an event along the strip due to resistive charge division. One may think of a particular event occuring within a pixel, defined by the strip and the vertical-position resolution of $900 \mu \mathrm{m}$ FWHM. The total number of pixels for the focal plane detector is $\sim 620$.

Positive identification of a particular reaction product may be accomplished by observing its decay chain if the decays of the daughter nuclides are known. A highenergy signal in the SSD, observed in coincidence with a signal from the PGAC, indicates that a fusion-evaporation residue has been implanted in the silicon detector. One may then look for decays occurring within the same pixel. By time-stamping each event, it is trivial to determine the half-life for a particular decay. Since the residues are implanted near the surface of the detector, the detection efficiency for subsequent decays is approximately $50 \%$. Due to the large number of pixels, the average time between implantations in a given pixel is on the order of seconds, thus permitting the decay chain to be observed through several generations. The decay-correlation technique is particularly well suited for work near the proton drip line where the halflives of the nuclides of interest are typically less than $50 \mathrm{~ms}$, making false coincidences unlikely. Unfortunately, this technique is not well suited for the region of the proton drip-line immediately below the $\mathrm{N}=82$ closed shell, since these nuclides decay primarily by electron capture rather than alpha emission.

\section{A SEARCH FOR LIGHT THALLIUM ISOTOPES}

In a first experiment to search for new proton emitters using the BGS, three ${ }^{78} \mathrm{Kr}$ bombardments of a $1.5 \mathrm{mg} / \mathrm{cm}^{2} 70 \%$-enriched ${ }^{102} \mathrm{Pd}$ target were performed at midtarget energies of 360,375 and $385 \mathrm{MeV}$. Analysis of the data from this experiment is still in progress, and all results presented herein should be considered preliminary. This paper will focus on the results of the $375 \mathrm{MeV}$ bombardment. The goal of this measurement was to reproduce the results of the earlier study of ${ }^{177} \mathrm{Tl}$ performed at Argonne National Laboratory, both to provide energy calibration points and to determine if the BGS was working as expected. Poli, et al.[3], produced ${ }^{177} \mathrm{Tl}$ in its ground state using the ${ }^{102} \mathrm{Pd}\left({ }^{78} \mathrm{Kr}, \mathrm{p} 2 \mathrm{n}\right)$ reaction at $370 \mathrm{MeV}$ with a cross section of 10 $\mathrm{nb}$; an isomer was also produced with a $30 \mathrm{nb}$ cross section. The ground state $\left[\mathrm{T}_{1 / 2}=\right.$ $67(37) \mathrm{ms}$ ] decayed by emission of either an $1156(20) \mathrm{keV}$ proton [27(13)\%] or a $6907(7) \mathrm{keV}$ alpha particle. The isomer decayed by emission of a $1958(10) \mathrm{keV}$ proton [51(8)\%] or a $7487(13) \mathrm{keV}$ alpha particle with a half life of $451(106) \mu \mathrm{s}$.

Figure 2 shows the alpha spectrum measured at the focal plane of BGS during a 17 hour $375 \mathrm{MeV}$ bombardment at an average beam current of approximately $10 \mathrm{pnA}$. Decay events were differentiated from implantations by requiring that they be anticoincident with signals from the PGAC; no other conditions were applied to generate 
this spectrum. The energy resolution for alpha decays was $70 \mathrm{keV}$. The peaks have been assigned to various $\mathrm{Hg}, \mathrm{Au}$ and $\mathrm{Pt}$ isotopes on the basis of their energies.

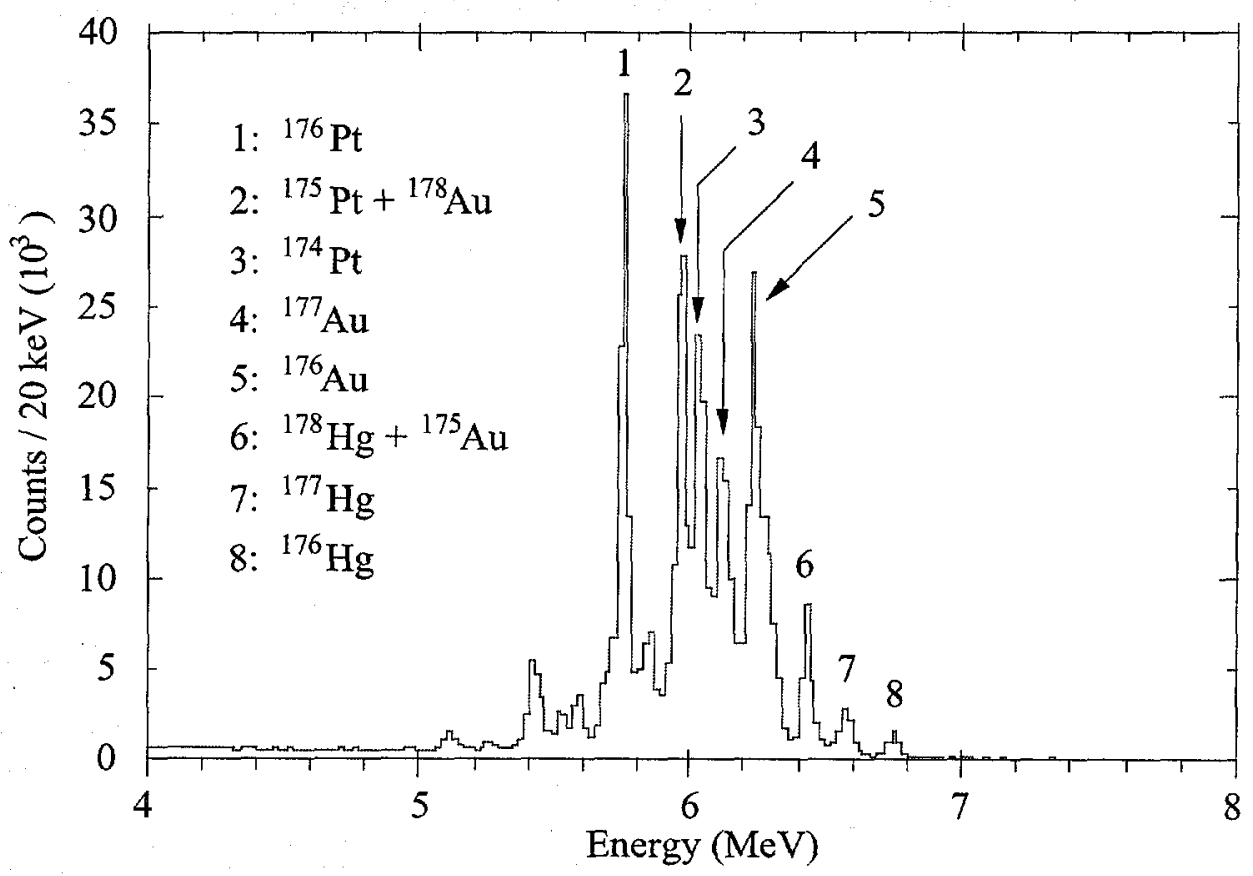

FIGURE 2. Alpha decays observed during the $375 \mathrm{MeV}{ }^{78} \mathrm{Kr}$ bombardment of ${ }^{102} \mathrm{Pd}$. All events shown were detected in anti-coincidence with signals from the PGAC. Preliminary assignments have been made for some of the peaks based on their energies.

When compared to the work of Poli and collaborators [3], one sees that the distribution of isotopes produced was slightly different. This may be due in part to the somewhat higher beam energy employed. Based on the yield of two key isotopes, ${ }^{177} \mathrm{Hg}$ and ${ }^{177} \mathrm{Au}$, it would appear that the transport efficiency of the BGS was significantly lower than had been expected. Several factors may have contributed. In an effort to increase the suppression of the primary beam at the focal plane, a stack of carbon degrader foils, with a total thickness that was varied from 1.09 to $0.76 \mathrm{mg} / \mathrm{cm}^{2}$, was placed immediately behind the target. This may have adversely affected the BGS optics and lowered transmission. Subsequent measurements suggest that these foils were not necessary to achieve adequate beam suppression. These foils also blocked the elastic scattering detectors used to measure beam current, so it is unclear how accurate the beam intensity cited above is. Finally, much of the time was spent attempting to optimize the tune of the separator. At the time of this measurement, the analysis code was still in development, which complicated tuning due to lack of on-line diagnostics. However, the yield of alpha emitters was within a factor of two of that measured using the Fragment Mass Analyzer at Argonne [3], and this result was achieved in a shorter time period ( 17 hours vs. 65 hours). 
In order to positively identify the various isotopes produced, correlations were sought between the implantation of evaporation residues and the subsequent chain of decays. A time stamp for each event was generated using independent $\mathrm{MHz}, \mathrm{kHz}$ and $\mathrm{Hz}$ clocks. During analysis, all events coincident with signals in the PGAC were assumed to be implantations and stored in a correlation buffer. When a decay event was observed, a search was made for earlier implants or decays within $0.9 \mathrm{~mm}$ on the same strip of the SSD. The maximum preceding time interval that was searched for correlations was determined by the energy of the event in accordance with typical half-lives; for alpha decays of greater than $6.7 \mathrm{MeV}$, this was set to $150 \mathrm{~ms}$. When correlations were found between alpha decays and earlier events, the decays were added to the correlation buffer as well.

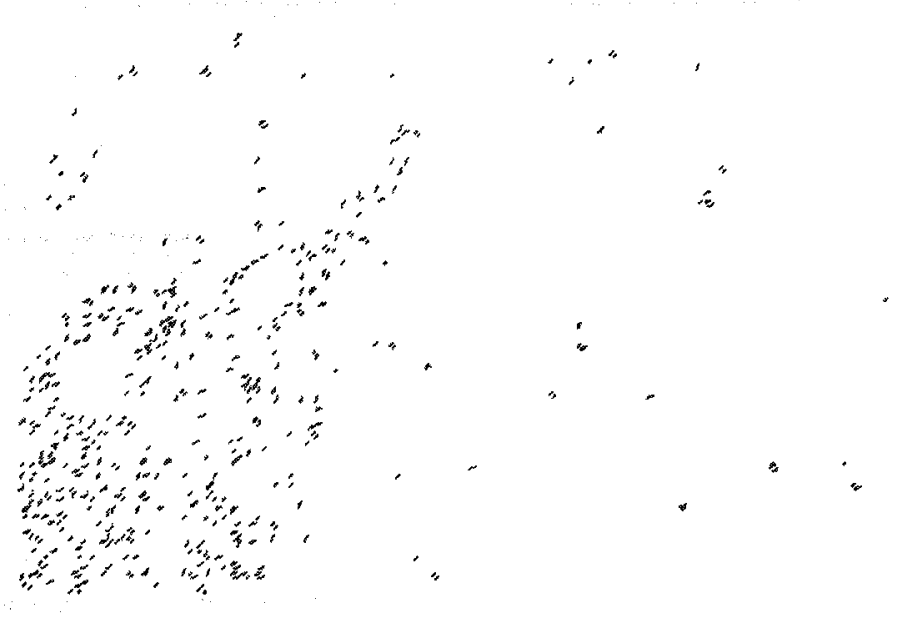

FIGURE 3. A parent-daughter alpha-decay correlation plot generated from the data collected during the $375 \mathrm{MeV}{ }^{78} \mathrm{Kr}$ bombardment. See text. The contour lines increase logarithmically; black indicates the 8-count countour.

Figure 3 shows a correlation plot between successive generations of alpha decays from the same data set that generated Fig. 2. The decay energy from the parent lies along the $\mathrm{x}$-axis and the subsequent decay is plotted on the y-axis. The circles, which 
decays. $\Lambda$ time stamp for each event was generated using independent $\mathrm{MHz}, \mathrm{mHz}$ and $\mathrm{Hz}$ clocks. During analysis, all events coincident with signals in the PGAC were assumed to be implantations and stored in a corrclation buffer. When a decay event was observed, a search was made for earlicr implants or decays within $0.9 \mathrm{~mm}$ on the same strip of the SSD. The maximum preceding time interval that was searched for correlations was determincd by the cnergy of the event in accordance with typical half-lives; for alpha decays of grcatcr than $6.7 \mathrm{MeV}$, this was set to $150 \mathrm{~ms}$. When correlations were found betwecn alpha decays and earlier events, the decays were added to the correlation buffer as well.

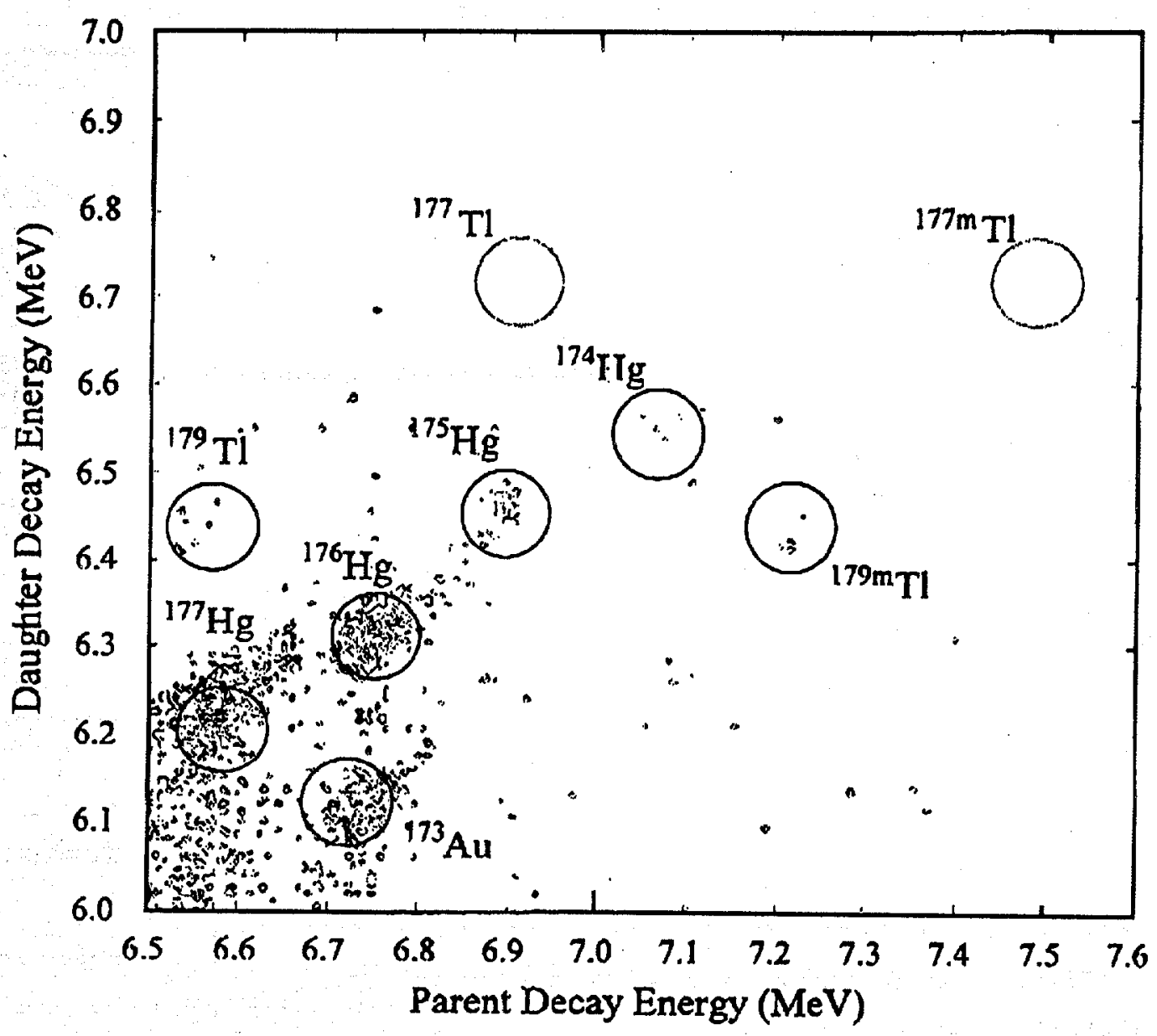

FIGURE 3. A parent-daughter alpha-decay correlation plot generated from the data collected during the $375 \mathrm{McV}{ }^{70} \mathrm{Kr}$ bombardment. See text.

Fig. 3 shows a correlation plot betwecn successive generations of alpha decays from the same dala set that generated Fig. 2. The decay energy from the parent lies along the $\mathrm{x}$-axis and the subscquent decay is plotted on the $y$-axis. The circles, which have a diameter encompassing $100 \mathrm{keV}$, indicate the positions of various parentdaughter decay series, as based on their literature decay energies [4]. Each is labelled with the parent isotope. The ${ }^{177} \mathrm{Hg}-{ }^{173} \mathrm{Pt},{ }^{173} \mathrm{Au}-{ }^{169} \mathrm{Tr},{ }^{176} \mathrm{Hg}-{ }^{172} \mathrm{Pt}$ and ${ }^{175} \mathrm{Hg}-{ }^{171} \mathrm{Pt}$ 


\section{OUTLOOK FOR THE WU'TURE}

These preliminary results are quite encouraging. However, this experiment also made it clear that the BGS, in its original configuration, had several shortcomings. In the months since, a variety of improvements have been planned and, in most cases, implemented. Figure 4 is provided to illustrate some of the problems with the original configuration of the separator and its detectors. It shows energy spectra measured in the SSD at the focal plane during the $360 \mathrm{MeV}{ }^{78} \mathrm{Kr}$ bombardment. The black line shows the sum of all events measured at the focal plane. The dark grey line shows the subset of those events which were coincident with signals from the PGAC, and thus interpreted as implantations of tusion-cvaporation residues. The light grey line shows events that were anticoincident with PGAC signals, and therefore interpreted as decays.

It is seen that the majority of events above $2 \mathrm{MeV}$ in Fig. 4 are coincident with the PGAC. However, between 5 and $7 \mathrm{McV}$ alpha decay signals dominate. Above, 7 $\mathrm{MeV}$, virtually no alpha decays should be observed, so it is clear that some implantation cvents arc passing through the PGAC without producing a signal. To improve the discrimination between implant- and decay-type events, a second $P C \wedge C$ was added to the BGS upstream of the focal plane shorlly after this experiment. This greatly improves the discrimination.

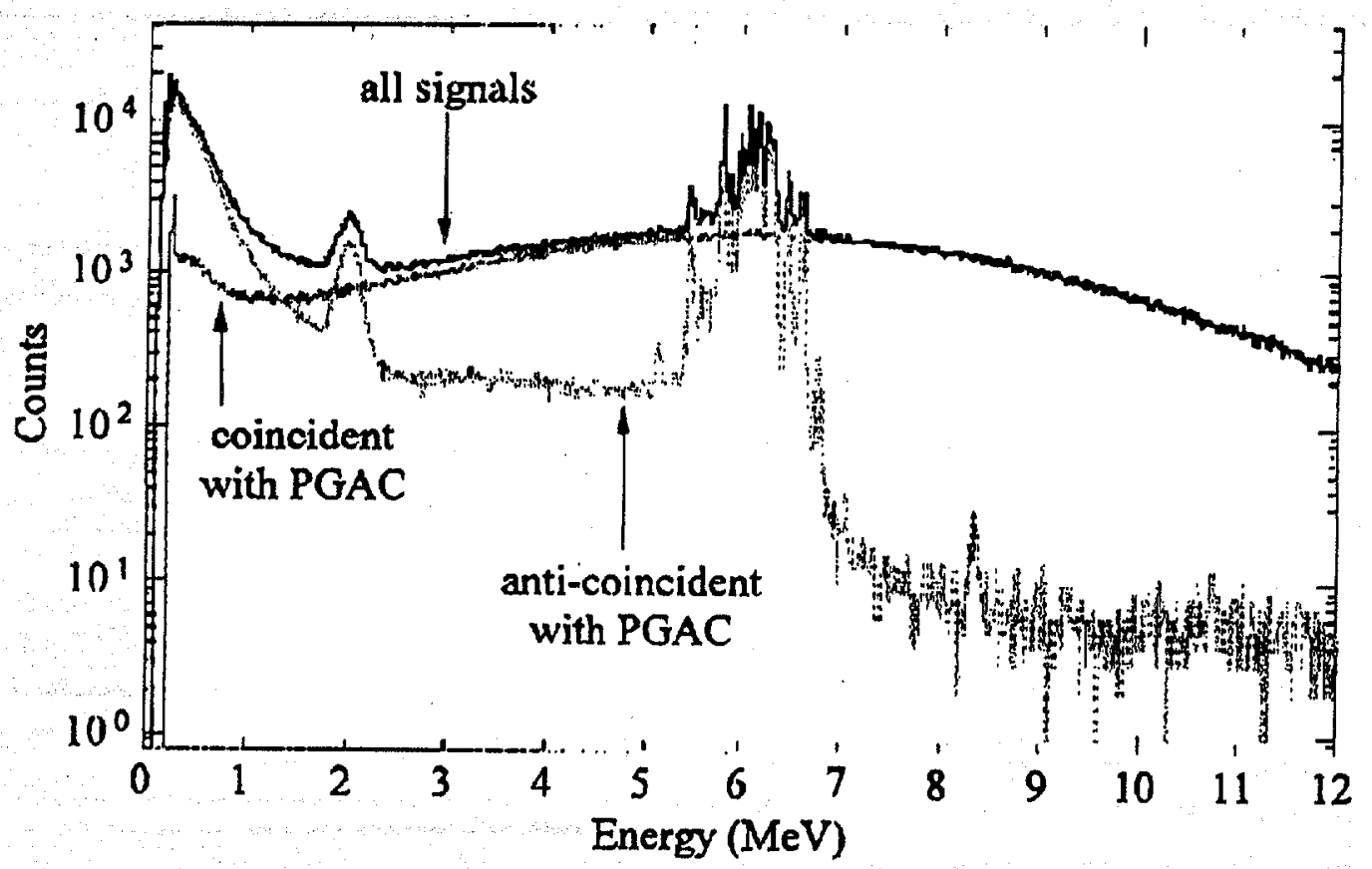

FIGURE 4. Energy spectra of events observed in the silicon strip detector (SSD) located at the BGS focal plane. The black line indicates the spectrum of all events. The heavy and light grey lincs indicate the subsets of those events which were either coincident or anticoincident with PGAC signals, respectively. 
$\Lambda$ broad peak which is mostly anticoincident with the PGAC is seen in the spectrum near $2 \mathrm{MeV}$. This is due to high-encrgy Hc ions resulting from collisions between heavy ions and the He gas in the BGS. Bccause they are high-energy and low-Z, they producc minimal signals in the PGAC and arc not stopped in the SSD. Soon after this experiment, a second silicon strip detector was put in place behind the focal plane SSD. Events observed in coincidence with signals $>1 \mathrm{MeV}$ from this detector are discarded. The small peak near $8 \mathrm{MeV}$ is of unknown origin, but may have been caused by a similar mechanism. It docs not seem to be correlated with alpha decay chains.

As noted earlicr, the cncrgy and position resolution of the SSD average approximately $70 \mathrm{keV}$ and $0.9 \mathrm{~mm}$, with some strips much worse than others. This was due to radiation damage sustained during the commissioning of the BGS. This was later improved somewhat by annealing the detector. It is planned that this SSD will be replaced early in 2000 by two $60 \mathrm{~mm} \times 60 \mathrm{~mm}$ 16-strip silicon detcctors placed side-by-side at the focal plane. Besides improving the energy and position resolution, this will also provide more focal plane coverage. The enhanced position sensitivity will increase the average time between implantations at a given position by a factor of approximately 8 , decreasing the chances of false coincidences betwcen different decay chains. This should permit more decay generations to be observed, or higher event rates to be tolerated.

Most of the decay events observed below $5 \mathrm{MeV}$ in Fig. 4 are duc to alpha particles which decay out of the detector, so that only a partial energy signal is observed. To improve this situation, six additional SSD's will be placed around the four sides of the focal plane SSD, perpendicular to its surfacc. Thesc will detect approximately $60 \%$ of alpha particles or protons that escape from the focal plane detector. In addition to increasing the odds of observing decay chains through multiple generations, this will reducc the "background" in the region between 1 and $2 \mathrm{MeV}$ where proton decays will be observed. These side detectors will also be installed in early 2000 .

During this experiment, the dead time of the data acquisition system after each event was about $200 \mu \mathrm{s}$. This would prevent the observation of isotopes with very short half-lives. The CAMAC-based system which was used is being replaced by a VME-based system, which should significantly reduce this problem. This is especially important due to the large number of additional channcls which will be needed for the new detectors described above. There have also becn improvements in the data analysis code that will improve on-line diagnostics.

Finally, experience with tuning the separator will also prove important. In particular, recent measurements have indicated that suppression of the primary beam is sufficient in this reaction without degrading the beam and recoils after the target. In Fig. 4, the evapotation residues have been degradcd to the same energy range as the alpha decays. Without the carbon degrader foils, the occasional implant event that does not produce a signal in the PGAC is unlikely to be mistaken for a decay. Also, the elastic scattering detectors bchind the target will no longer be blocked by degrader foils, so the beam intensity may be accurately assessed.

If necessary, we plan to repeat this experiment in order to take advantage of these improvements to the BGS. An addition, several other measurements of proton and alpha-particle cmittcrs in this region of the Chart of the Nuclides are planned. These 
studies will provide important decay, mass and structurc information about nuclej at. the extreme limits of stability.

\section{CONCLUSION}

Although yields observed in this early BGS experiment were not as high as had been expected, the results of the preliminary analysis of the $375 \mathrm{McV}$ data are intriguing. Much more work still remains to be done on the analysis at this time; only about half of the $375 \mathrm{MeV}$ data has been examined. Improvements in the correlation algorythms may also yield additional results which wcrc misscd. Analysis of the data taken at $360 \mathrm{MeV}$ and $385 \mathrm{MeV}$ has only been very cursory at this point.

Partially as a result of this experiment, a series of improvements to the BGS are being implemented. Many of these improvements wcre in place during the element 118 measurements [7], and proved to be critical to thc success of those experiments. The present study has indicated that the BGS will be an excellent tool for the investigation of proton drip-line nuclei ncar the $Z=82$ shell closure. In particular, its high transport cfficiency and the ability to withstand high beam intensitics will permit isotopes produced in very low yjelds to be ubserved and identified. This will be especially true once the planned improvoments are in place.

\section{ACKNOWLEDGMENTS}

We wish to acknowledge the excellent work of David Ruiz and the rest of the 88 " Cyclotron machine shop in building and installing the BGS. We also thank Sigurd Hofinann and GST for the silicon strip detcctor used in this measurement, and the 88" Cyclutrun opcrations staff for assisting with this experinent. This work was supported by the U. S. Department of Encrgy under contracts DE-AC03-76SF00098 (Lawrence Berkclcy National Laboratory), DE-AC05-960R22464 (Oak Ridge National Laboratory, managed by I ockheed Martin Fnergy Research Corporation), DE-FG0296ER40983 (University of Tennessee), and DE-FG02-96ER40978 (Louisiana Slate University).

\section{REFERENCES}

1. Cerny, J., Estcrl, J. E., Gough. R. A. and Sextro, R. G.. Phys. Letters 33B, 284-286 (1970).

2. Woods, P. S., and Davids, C. N., "Nuclei Beyond the Proton Drip-Line," in Ann. Rev. Nucl. Part. Sci., 47, 541-90 (1997).

3. Poli, C. L., et al. Phys. Rev. C 59, R2979-R2983 (1999).

4. Pfennig. G., Klewe-Nebenius, H., Seelmann-Eggebcrt, W., Karlsruher Chart of the Nuclides, $6^{\text {th }}$ ed. revised reprint, Institute fuer Instrumentclle Analytik, 1998.

5. Uusitalo, J., et al., Z. Phys. A 358, 375-376 (1997).

6. Sewerynizk, D., el al., Phys. Rev. C 60, 031304-1 ... 031304-4 (1999).

7. Ninov, V. et al., Phys. Rev. Lett. 83. 11 04-7 (1999). 